\title{
Large cell neuro endocrine lung carcinoma-many questions
}

\begin{abstract}
Pulmonary large cell neuroendocrine carcinomas (LCNECs) are rare tumors of the lung. The diagnosis is based on the presence of both neuroendocrine morphology as well as immunohistochemical evidence of neuroendocrine markers. They express high grade features. In the 2015 World Health Organization classification, LCNEC was considered as part of the pulmonary neuroendocrine carcinomas. The clinical presentation and natural history of LCNEC appear similar to the other high-grade neuroendocrine pulmonary tumors. According to most studies, survival appears worse for LCNEC when compared to non-small cell carcinoma (NSCLC) and other large cell carcinomas (LCCs), but similar to that of small cell lung carcinoma (SCLC).
\end{abstract}

Volume 9 Issue 4 - 2018

\author{
Hajj Adel Anis \\ Cedars - Jebel Ali International Hospital, UAE
}

Correspondence: Hajj Adel Anis, Medical Oncologist at Cedars - Jebel Ali International Hospital, 9370 Rue Lajeunesse, Montreal, UAE, Tel 438992 55I6, Email ahajj@dr.com

Received: June 27, 2018 | Published: July 27, 2018

Keywords: world health organization, regarding immunohistochemistry analysis, neuron-specific enolase

Abbreviations: WHO, world health organization; LCCs, large cell carcinomas; NSCLC, non-small cell carcinoma; LCNECs, large cell neuroendocrine carcinomas; $\mathrm{AC}$, adenocarcinoma

\section{Facets of LCNEC diagnosis}

Even amongst expert pathologists, difficulties are encountered when making a diagnosis of LCNEC. The World Health Organization (WHO) suggested tolimit/avoid the diagnosis of LCNEC on cytology/ small biopsy. Pathologic characterization may sometimes show coexisting foci of squamous or adenomatous differentiation in these tumors, creating mixed pathologic entities called "mixed LCNEC". LCNEC and adenocarcinoma (AC) share some pathologic features, such as growth patterns and necrosis, so, differential diagnosis may be challenging. Indeed, a mitotic rate of 11 mitoses or more per 10 high-power fields is a key factor to differentiate LCNEC from AC. To achieve a more precise diagnosis, a careful pathologic review is recommended because it is quite easy to mistake an LCNEC for a poorly differentiated NSCLC, an AC and even an SCLC.

Regarding immunohistochemistry analysis, pulmonary LCNECs express typical neuroendocrine markers such as chromogranin, neuron-specific enolase, synaptophysin and somatostatin, which are necessary to obtain the diagnosis. Pulmonary LCNEC proliferative rate is higher than classic LCC and other low-grade neuroendocrine tumors, such as carcinoids. LCNEC shows higher expression of $\mathrm{Ki}-$ $67, \mathrm{Bcl}-2$, p21 and of telomerase activity; abnormal p53, absent Rb. Because of important genomic based similarities between LCNEC and SCLC, it was suggested to combine immunohistochemical and genomic analysis to differentiate each other.

\section{Treatment challenges}

There is no standard treatment of pulmonary LCNEC. Unfortunately, the majority of pulmonary LCNEC are not eligible for primary surgical resection because of local or systemic spread. However, this approach when feasible constitutes the principal way to obtain an accurate diagnosis. Moreover, it may improve survival in the absence of mediastinal lymphnode metastases. Optimization of perioperative treatments could improve outcome. Neoadjuvant or adjuvant chemotherapeutic treatment may represent reasonable options to prevent disease relapse. The optimum regimen has not been established yet.

Currently, a phase III trial of adjuvant cisplatin plus irinotecan versus etoposide has been designed and is still ongoing in Japan. A defined biomarker of response to chemotherapy has been not yet identified. The role of radiotherapy is still unclear, but some authors suggest its use in locally advanced disease setting. Prophylactic cranial irradiation is actually not recommended in pulmonary LCNEC patients.

There is no consensus on standard treatment for recurrent or advanced LCNEC. Positive results with SCLC-based regimens in perioperative setting encouraged to use this strategy also in unresectable disease. Efforts are steadily directed to clarify whether pulmonary LCNEC could be treated as SCLC, as NSCLC or as another variant of lung tumor. LCNEC expresses histological characteristics which overlap with NSCLC, and occasionally, with SCLC, thereby, it is considered as a subtype of NSCLC and is typically treated with chemotherapy. In the current European Society for Medical Oncology (ESMO) guidelines for NSCLC, no specific treatment for LCNEC is described. In the American Society of Clinical Oncology (ASCO) guidelines, either platinum-etoposide chemotherapy treatment (SCLC-Type) or the same regimen as for nonsmall cell-non squamous carcinoma being the combination of platinum with gemcitabine, are advised for LCNEC. However, SCLC-Type chemotherapy is considered by expert opinion to be most appropriate and several observations suggest that LCNEC should respond best to an SCLCType chemotherapy treatment. ${ }^{1-5}$

\section{Conclusion}

Few data are available on biological treatment in pulmonary LCNEC. Angiogenesis is known to be one of the greater mechanisms of tumor evolution; therefore, an important role could be played by inhibition of angiogenesis pathways. Under clinical development, new agent nedaplatin, a platinum-based antineoplastic drug, is studied in combination with irinotecan. Future therapeutic targets could be represented by tropomyosin-related kinase $\mathrm{B}$ and brain-derived neurotrophic factor that are highly expressed in LCNEC as markers of invasiveness. Additional large cohort studies on the biology of 
high-grade neuroendocrine carcinomas are warranted and will aid establishing novel approaches to clinical managements of patients with LCNEC.

\section{Acknowledgements}

None.

\section{Conflict of interest}

The author declares no conflict of interest.

\section{References}

1. Battafarano RJ, Fernandez FG, Ritter J, et al. Large cell neuroendocrine carcinoma: an aggressive form of non-small cell lung cancer. $J$ Thorac Cardiovasc Surg. 2005; 130(1):166-172.
2. Paci M, Cavazza A, Annessi V, et al. Large cell neuroendocrine carcinoma of the lung: a 10year clinicopathologic retrospective study. Ann Thorac Surg. 2004; 77(4):1163-1167.

3. Jiang SX, Kameya T, Shoji M, et al. Large cell neuroendocrine carcinoma of the lung: a histologic and immunohistochemical study of 22 cases. $\mathrm{Am}$ J Surg Pathol. 1998;22(5):526-537.

4. Iyoda A, Hiroshima K, Nakatani Y, et al. Pulmonary large cell neuroendocrine carcinoma: its place in the spectrum of pulmonary carcinoma. Ann Thorac Surg. 2007;84(2):702-707.

5. Iyoda A, Battafarano RJ. Large-cell neuroendocrine carcinoma of the lung: an aggressive neuroendocrine lung cancer. Ann Thorac Surg. 2007;18(3):206-210. 